\title{
3D CORE CALCULATION BASED ON THE METHOD OF DYNAMIC HOMOGENIZATION
}

\author{
Antonio Galia, Igor Zmijarevic and Richard Sanchez* \\ DEN-Service d'études des réacteurs et de mathématiques appliquées (SERMA), \\ CEA, Université Paris-Saclay, F-91191, Gif-sur-Yvette, France \\ antonio.galia@cea.fr, igor.zmijarevic@cea.fr, richard.sanchez@cea.fr
}

\begin{abstract}
The classical two-step calculation scheme has been extensively used to perform threedimensional deterministic core calculations thanks to its fast results. On the other hand, direct 3D transport calculations and 2D/1D Fusion methods, mostly based on the method of characteristics, have recently been applied showing a prohibitive computational time for routine design purposes as well as in the context of multiphysics and core depletion calculations, due to current machine capabilities.

The Dynamic Homogenization method is here proposed as an alternative technique that may lie between the classical and the direct approaches in terms of precision and performance. In this work, the method is applied to the NEA "PWR MOX/UO2 Core Benchmark" for a 3D configuration. Comparison of pin power relative errors and computational cost against the two-step and direct approaches are presented.
\end{abstract}

KEYWORDS: Core Calculations, Dynamic Homogenization, Domain Decomposition, PWR, APOLLO3 ${ }^{\circledR}$

\section{INTRODUCTION}

The two-step calculation scheme has been widely used for core modeling and design because of the main advantage of obtaining fast results for different core configurations. The first step of this approach consists of "off-line" 2D calculations for each assembly type, that are typically performed with the method of characteristics (MOC) on a fine spatial and energy mesh with high order angular discretization, to produce a library of homogenized cross sections that are calculated for different physical parameters, such as burnup, moderator density, fuel temperature, etc. At this stage, the surrounding environment of the assembly is not known, so conservative conditions are imposed at the boundaries as if the motif was repeated to infinity (the so-called infinite lattice calculation). The $k_{\text {eff }}$ of the assembly can then be very different from one, so the weighting spectrum is corrected by introducing a critical-leakage model, where the leakage coefficient and the buckling of the homogenized medium $\left(D B^{2}\right)$ are searched iteratively in such a way that the multiplication constant of the assembly is equal to one. The flux resulting from the heterogeneous transport equation with imposed critical leakage, is finally used to homogenize the cross-sections. Nevertheless, a different approach has to be used for reflector homogenization, since no multiplication occurs

\footnotetext{
*permanent adress: richard.abuli@ protonmail.com
} 
and vacuum conditions are imposed on at least one boundary. Typically, a separate calculation is performed in a smaller motif to produce homogenized data that are subsequently used for the whole reflector surrounding the core. Once the cross-sections have been homogenized in space and in energy, an equivalence technique between the fine-transport and the low-order operators is generally applied. The most common techniques are the SuPerHomogenization (SPH) $[1,2]$ and the Flux Discontinuity Factors (FDF) [3-6]. For 2D configurations it has been shown that using equivalence parameters improves significantly the quality of the solution of the two-group diffusion core calculation [5,7], and that typical pin power errors found in this literature are reduced from around $10-12 \%$ to roughly $5-7 \%$ with respect to a reference calculation of a typical LWR at nominal conditions and. The main source of these errors is due to the fact that the reference problem chosen to produce homogenized transport data does not take into account the environment. The assemblies that are the most sensitive to this approximation are those at the peripheral area next to the reflector and those next to other types of assembly such as the UOx-MOx, Rodded-Unrodded interfaces. Another source of errors arises because the coarse discretization of the two-group diffusion operator is inadequate to describe the physics of the problem, making the simplified $P_{3}$ operator more attractive. To overcome the first problem, many attempts were made in the past in order to consider the neighboring assemblies at the homogenization stage. For instance, a straightforward approach involves the calculation of several colorsets, requiring the computational cost of many combinations of assembly types. Another approach is to approximate the environment effect by correcting the homogenized nuclear data with a linear perturbative method [8,9], where no extra lattice calculation needs to be performed. Recently, a modern calculation scheme was explored by Takeda et al. [10]. It consists of an iterative process between core and lattice calculation, where the core macro currents are used to compute the albedo boundary conditions that are imposed at the assembly level in the next iteration. This approach is computationally more expensive but it does not require any off-line branch calculations. However, each lattice calculation is independent of the others because it only depends on core quantities, which entails that the spectrum of the neighboring assemblies cannot be properly taken into account. The method that we propose takes the exact fine distribution in energy, space and angle from the neighboring assemblies, which is then normalized by the partial core macro-current, so as to preserve the macroscopic assembly core exchanges. Each assembly has then different boundary conditions that account for its position in the core and for the isotopic content of the neighboring assemblies: both sources of gradients in a reactor. The method was proposed by P. Mondot and R. Sanchez in 2003 [11] and tested for simple cases in $1 \mathrm{D}$ problems. In 2014, D. Colameco et al. extended the application to $2 \mathrm{D}$ configurations, introducing in the iterative process an equivalence by discontinuity factors [12]. They obtained good agreement against reference transport calculation for a $2 \times 2$ cluster in 2D of UOx and MOx assemblies with reflection boundary conditions. The method was also adopted for Pebble Bed Reactors by M. Grimod et al. in 2015 [13], with a further approximation on the interface exchange: an isotropic angular flux to avoid angular dependencies. In a recent work [14], we have tested the method cor a PWR two-dimensional core with different types of assemblies at different burnups, where the solution showed results of better quality with respect to the classical two-step approach, eliminating the typical error peaks at the interface between different assembly types and improving the radial reflector response, where typically two-step fails. In terms of performance, the computational cost of the method applied to a $2 \mathrm{D}$ configuration was $25 \%$ less than a direct transport 2D calculation. All these methods, including the present one, are based on cross-section homogenization from 2D transport calculations. In this work, the method is applied to a 3D core, where an approximation of the axial leakage is introduced to couple the 2D layer fluxes. Finally, 
a comparison with direct transport (considered as reference) and the classical two-step approach is presented.

\section{3D DYNAMIC HOMOGENIZATION}

The Dynamic Homogenization (DH) is an iterative process between assembly and core calculations. Each assembly calculation is solved in 2D fine-transport with imposed multiplication constant $\lambda$, and fixed incoming boundary sources $\psi_{i n, i}$. The $\lambda$, being an integral parameter of the whole domain, is determined at the core level using Eq. (1):

$$
\lambda_{C}^{k+1}=\lambda_{C}^{k} \frac{\left\langle w, F_{C} \Phi^{k+1}\right\rangle}{\left\langle w, F_{C} \Phi^{k}\right\rangle},
$$

where $C$ stands for coarse, $F$ for production operator, $\Phi$ for scalar flux, $w$ for a weight function and $k$ is the iteration index of the outer coarse core iteration.

Even if the iterative calculation scheme is quite similar to a direct transport calculation (see Fig. 1), the angular fluxes are not continuous at the boundaries and the discontinuity is introduced by the ratio of coarse-to-transport partial currents. The idea is to impose the incoming flux as angular surface source with a macroscopic intensity determined from the core calculation. The incoming angular flux $\psi_{i n}$ is then equal to the product of the flux $\psi_{\text {out }}$ leaving the neighboring assemblies at the previous iteration, which gives the fine distribution in space, energy and angle, and a current normalization factor as in Eq. (2):

$$
\left.\psi_{\text {in }}^{g}(\mathbf{r}, \boldsymbol{\Omega})\right|_{\Gamma_{i j}} ^{n+1}=\left.\psi_{\text {out }}^{g}(\mathbf{r}, \Omega)\right|_{\Gamma_{i j}} ^{n} \times \frac{\left.J_{\text {in }}^{G}(\mathbf{r})\right|_{\text {core }} ^{n}}{\left.\sum_{g \in G} \int|\mathbf{n} \cdot \Omega| \psi_{\text {out }}^{g}(\mathbf{r}, \Omega) d \Omega\right|_{\Gamma_{i j}} ^{n}} \quad \forall g \in G,
$$

where $n$ denotes the global transport iterations on the interface currents, $\mathbf{n}$ is the unit vector normal to the interface and $g$ and $G$ are the fine (transport) and coarse groups. Note that the incoming fluxes are normalized so as to preserve the macroscopic coarse currents $J_{i n}$ entering the assemblies and averaged along the z-axis. The current normalization factor is typically not equal to one at the convergence of the iterative process: this introduces discontinuities at the interfaces that allow to preserve the eigenvalue from the low-order operator. Moreover, one may use a coarser description for the incoming transport flux. In order to couple the transport calculations performed at different axial layers we introduce the 2D-transverse Eq. (3) by applying the transverse operator $T_{z} \circ=\frac{1}{\Delta z_{k}} \int_{z_{k^{-}}}^{z_{k^{+}}} d z \circ$ to the $3 \mathrm{D}$ transport equation:

$$
\begin{gathered}
\left(\boldsymbol{\Omega} \cdot \nabla_{\perp}+\Sigma\right) \psi_{\perp, k}=q_{\perp, k}+\mathrm{L}_{z_{k}} \\
\mathrm{~L}_{z_{k}}\left(\mathbf{r}_{\perp}, \boldsymbol{\Omega}\right)=\frac{1}{\Delta z_{k}} \int_{z_{k^{-}}}^{z_{k^{+}}} \mu \frac{\partial \psi\left(\mathbf{r}_{\perp}, z, \boldsymbol{\Omega}\right)}{\partial z} d z=\left.\frac{1}{\Delta z_{k}} \mu \psi\left(\mathbf{r}_{\perp}, z, \boldsymbol{\Omega}\right)\right|_{z_{k^{-}}} ^{z_{k^{+}}}
\end{gathered}
$$

Here $\Delta z_{k}=z_{k+}-z_{k-}$ is the thickness of axial layer $k$. Note that the group index has been omitted, $\nabla_{\perp}=\frac{\partial}{\partial x}+\frac{\partial}{\partial y}, \psi_{\perp}=\psi\left(\mathbf{r}_{\perp},.\right)$, and the axial leakage $\mathrm{L}_{z_{k}}$ is expressed as a volumetric source term and defined as Eq. (4), where $\mu$ is the axial cosine. The first approximation that we adopted is to symmetrize the leakage source with respect to radial plane, in order to have an axially symmetric 2D fine-transport problem, which gives the advantage to treat half the number of directions and halves the number of angular moments for the flux and the source expansions. This leads to the 
following approximated form for the leakage term:

$$
\mathrm{L}_{z_{k}}\left(\mathbf{r}_{\perp}, \mathbf{\Omega}\right)=\frac{1}{2 \Delta z_{k}} \mu\left(\psi\left(\mathbf{r}_{\perp}, z, \mathbf{\Omega}_{\perp}, \mu\right)-\left.\psi\left(\mathbf{r}_{\perp}, z, \mathbf{\Omega}_{\perp},-\mu\right)\right|_{z_{k^{-}}} ^{z_{k^{+}}}, \mu>0\right.
$$

Another approximation is introduced for the evaluation of the angular flux on the top and bottom of an axial layer. Typically this is done by a 1D calculation performed in transport (Fusion) or by a coarse operator (Hybrid Fusion), but we wanted to avoid this extra cost, by using the information provided by the 3D diffusion solver. Similarly to Eq. (2), we introduce Eq. (6) so as to preserve the total axial leakage of the core solution, where $J_{z_{k} \pm}^{ \pm, C}$ are respectively the right (+) and the left (-) partial core currents, and $J_{\perp, k}^{+}$is the partial axial transport current calculated using the $2 \mathrm{D}$ angular flux.

$$
\psi\left(\mathbf{r}_{\perp}, z_{k^{ \pm}}, \boldsymbol{\Omega}_{\perp}, \pm \mu\right) \approx \psi_{\perp, k}\left(\boldsymbol{\Omega}_{\perp}, \pm \mu\right) \times \frac{J_{z_{k^{ \pm}}}^{ \pm, C}}{J_{\perp, k}^{+}}, \mu>0
$$

The convergence test of Fig. 1 checks the following quantities and criteria for two successive global iterations $(n, n+1)$ :

- Eigenvalue $\lambda_{C}$ : $\left|\lambda_{C}^{n}-\lambda_{C}^{n+1}\right|<10^{-5}$

- Fission Integral $F_{r}$ per transport region $r: \max \left(\left|1-\frac{F_{r}^{n}}{F_{r}^{n+1}}\right|\right)<10^{-4}$;

- Incoming Flux $\psi_{s, d, g}^{-}$per surface element $s$, direction $d$ and group $g$ : $\left\|1-\frac{\psi_{s, d, g}^{-, n}}{\psi_{s, d, g}^{-, n+1}}\right\|_{2}<10^{-4}$;

- Axial Leakage $\mathrm{L}_{z_{k}, r, d, g}$ per transport region $r$, direction $d$ and group $g$ : $\left\|1-\frac{L_{z_{k}, r, d, g}^{n}}{\mathrm{~L}_{z_{k}, r, d, g}^{n+1}}\right\|_{2}<10^{-3}$.

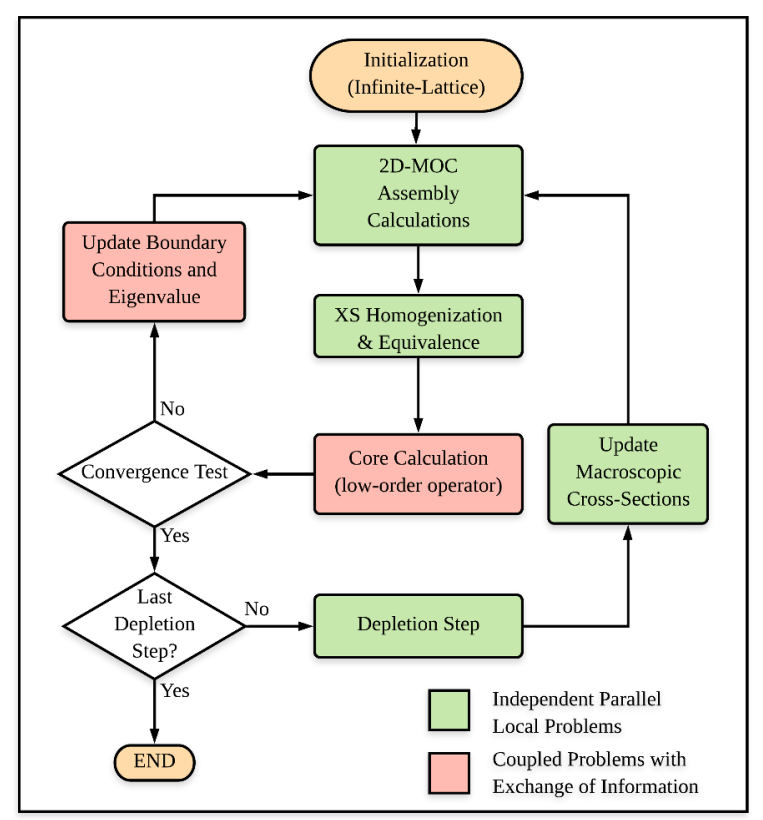

Figure 1: Dynamic Homogenization calculation scheme.

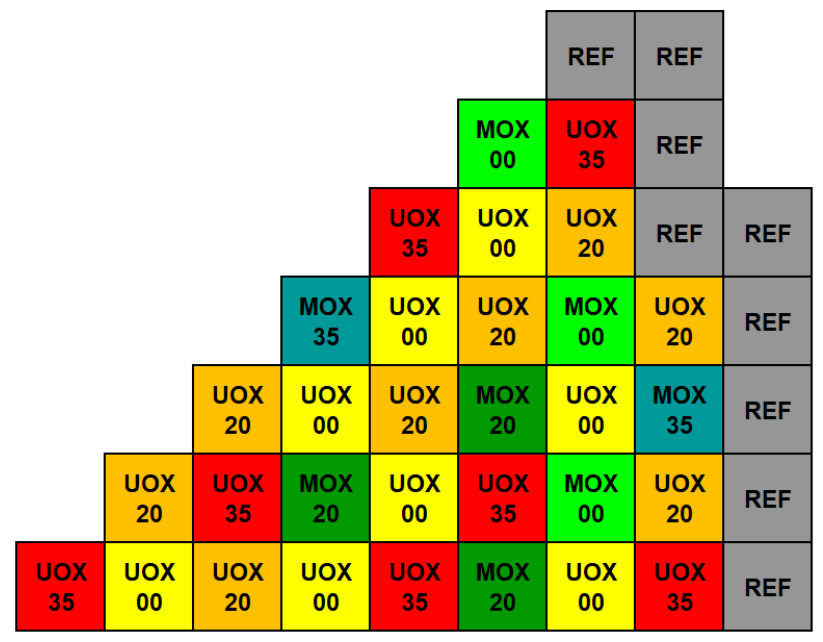

Figure 2: Core layout of NEA Benchmark with 1/8 radial symmetry [15]. 


\section{APPLICATION AND COMPARISON}

In order to compare the classical and the DH approaches, we analized a problem inspired by the NEA PWR MOX/UO2 Core Transient Benchmark [15], adopting some simplifications in order to have a smaller data library to process for preliminary calculations. We considered two types of assemblies: the UOx with $4.5 \%$ enrichment and the MOx with an average content of Pu-fissile of 4.3\%. Both assemblies have been irradiated at infinite-lattice conditions to obtain the necessary self-shielded macroscopic cross-sections at three burnup states: fresh fuel at $0 \mathrm{GWd} / \mathrm{t}$, once-burned at $20 \mathrm{GWd} / \mathrm{t}$ and twice-burned at $35 \mathrm{GWd} / \mathrm{t}$. The core layout is showed in Fig. 2. The UOx assemblies contain guide tubes, fuel pins with and without IFBA (Integral Fuel Burnable Absorber). The MOx assemblies contain guide tubes with and without WABA pins (Wet Annular Burnable Absorber), and a three-zone MOx fuel with $5.0 \%, 3.0 \%$ and $2.5 \%$ fissile $\mathrm{Pu}$ (in respectively the interior, periphery and corner positions). The active fuel lenght is $365.76 \mathrm{~cm}$ and the axial reflector is simplified with an axial water layer of $20.042 \mathrm{~cm}$ above and below the core. Nominal Power and isothermal conditions have been considered to evaluate nuclear data.

The direct and per assembly transport calculations have been performed using the IDT standalone solver of the code APOLLO3 ${ }^{\circledR}$ developed at CEA, which is a multigroup discrete ordinates short characteristics solver. The source iterations are carried out by cell sweeping, and the Coarse Mesh Finite Differences method (CMFD) is used as acceleration for the outer iterations and the Boundary Projection Acceleration (BPA) for the inners [16-18]. For two-step and DH approaches, a parabolic Nodal Expansion Method for diffusion (NEM) [7] has been used as coarse core operator. The fine energy mesh for transport has 172 energy groups with transport corrected $P_{0}$ scattering, and a level-symmetric quadrature formula $S_{8}$ was used for the angular variable. The number of 3D transport regions in the reference calculation was about 7.5 millions, whose axial height of each was $2 \mathrm{~cm}$. The pin-wise homogenization from 172 to 2 groups is done using a volume-flux weighting function and flux discontinuity factors (FDF) are applied as equivalence between transport and diffusion. The reflector cross-sections in the two-step scheme are homogenized into two media (one for the iron and one for the water) with a separate calculation of a 9x1 cluster of assemblies, and were used for the whole reflector surrounding the core.

In the DH approach instead, the reflector cross-sections were homogenized into a 17x17 grid geometry. However, we observed that an axially constant cross-section obtained by the 2D axial reflector homogenization, is not a good approximation, probably because the spectrum at the fuel-reflector interface is very different from the spectrum close to vacuum boundary, and the axial leakage is dominant. Because of this, at the interface of the axial reflector we obtained a peak of the power error around $18 \%$ with respect to the direct reference calculation. In order to improve the homogenized cross-sections in this region of the core, we have then redefine the local homogenization problem with a 1D axial calculation obtained by transverse integration of the 3D transport equation, that is solved per fuel assembly with imposed core eigenvalue and the fixed radial isotropic leakage source. Thanks to the 1D multigroup flux, we could homogenize the axial reflector so as to obtain radially constant but axially dependent cross-sections, and the associated axial discontinuity factors, reducing then the peak of power error to $9 \%$. Figure 3 shows the maximum power relative error for each radial plane and its RMS versus $z$, for two-step, DH with 2D axial reflector homogenization and DH with 1D axial reflector homogenization. For the three cases, flux discontinuity factors were applied. We can clearly observe that the quality of the DH solutions is improved with respect to the two-step scheme, with excellent agreement in the interior part of the core between $\mathrm{DH}$ and direct reference 3D transport calculation, and maximum discrepancies at the 
axial reflector interfaces, where DH and two-step behave in the same way. This can be explained by the fact that diffusion is not a good approximation at material discontinuities and, in both cases, no axial discontinuities in the fuel assemblies can be applied. It seems, that in DH only the first 20 $\mathrm{cm}$ of the core at the top and bottom are affected by the approximations of the model. Moreover, the two-step model has radially higher errors, especially at the interfaces of assemblies of different types and in the peripheral region next to the radial reflector, as a consequence of the wrong condition of fundamental mode in the reference homogenization problem. This is shown in Fig. 4 where we plotted the RMS of the axial relative error for the three cases. Table 1 shows the calculation run-time and the global RMS of the power relative error. As expected, DH lies between the direct transport and the two-step approaches in terms of accuracy and computational cost, reducing the global RMS from $2.1 \%$ to $1.1 \%$.

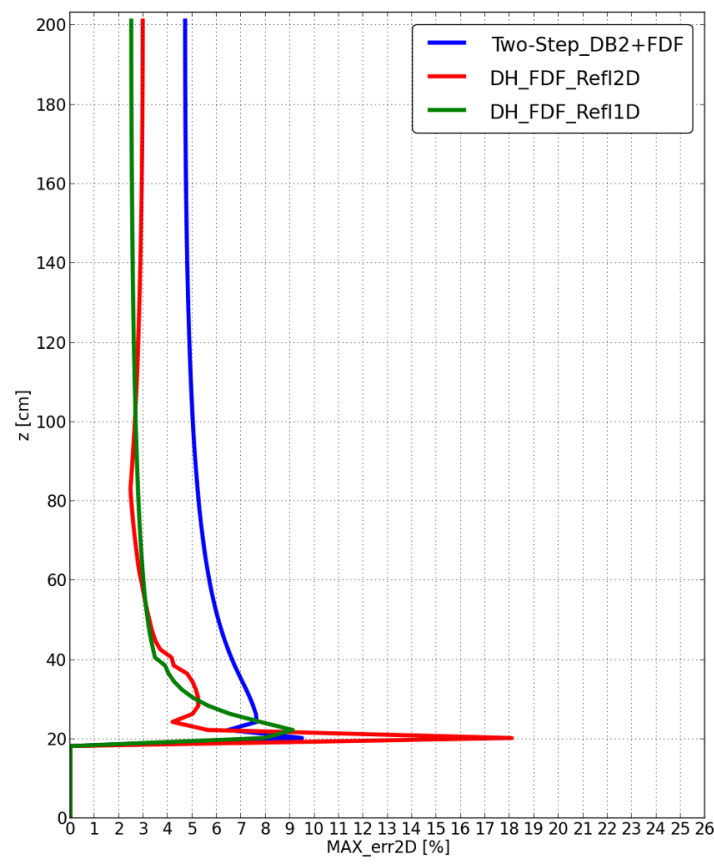

(a) MAX

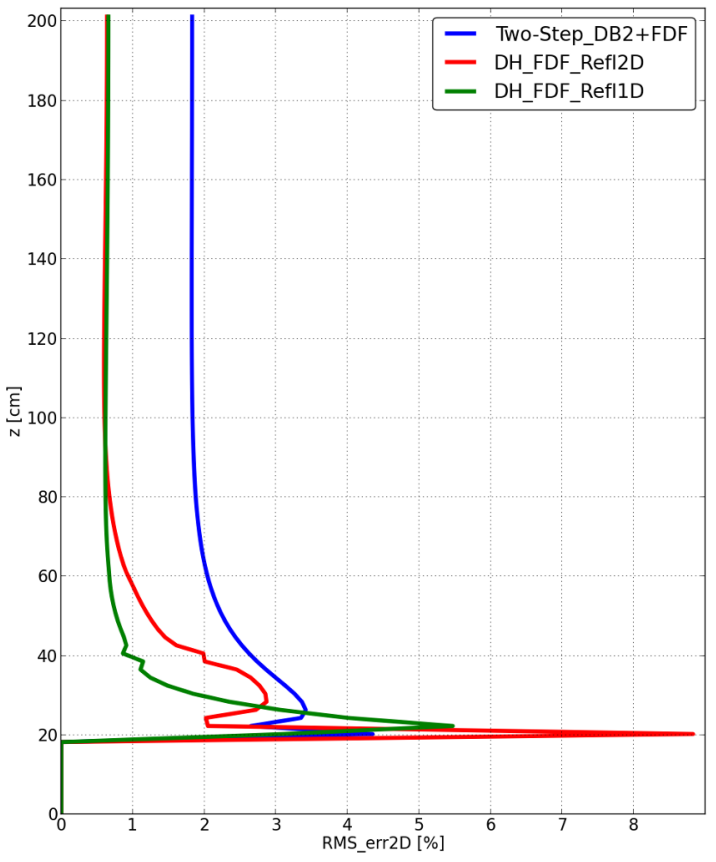

(b) RMS

Figure 3: Absolute MAX and RMS of power relative error per radial plane as a function of $z$ for the bottom part of the core.

\section{CONCLUSIONS}

The method of Dynamic Homogenization has showed good agreement with reference calculation, and only at the first $20 \mathrm{~cm}$ on top and bottom of the core high discrepancies appear with a peak of $9 \%$. This is caused by the fact that the approximation that we introduced for the axial leakage in the $2 \mathrm{D}$ transport calculation, may not be sufficient to describe the actual behavior at the interface with the axial reflector, since the problem is strongly axial and leakage is dominant. Globally, the RMS of the pin power error is reduced to $1.1 \%$ from $2.1 \%$ for the two-step calculation scheme, and the computational cost with respect to the reference direct 3D transport calculation is 25 minutes instead of 7.6 hours. 


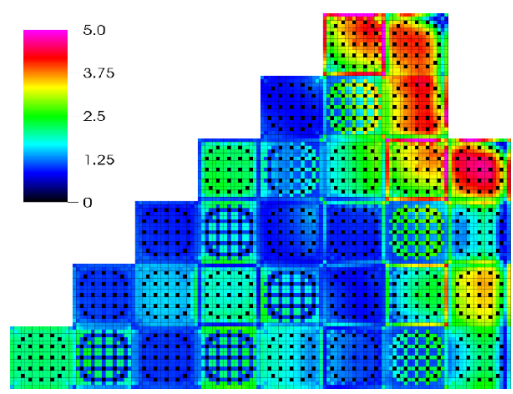

(a) Two-step

$\left(\max _{R M S}=5.37\right)$

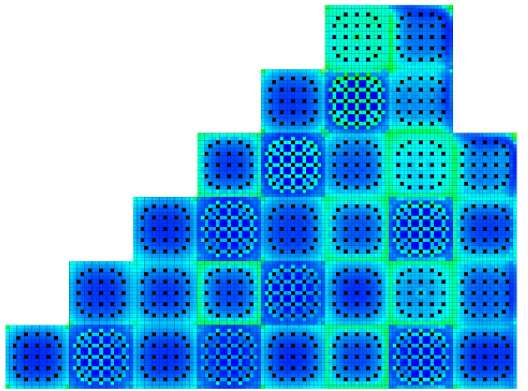

(b) DH with Refl2D

$\left(\max _{R M S}=2.9\right)$

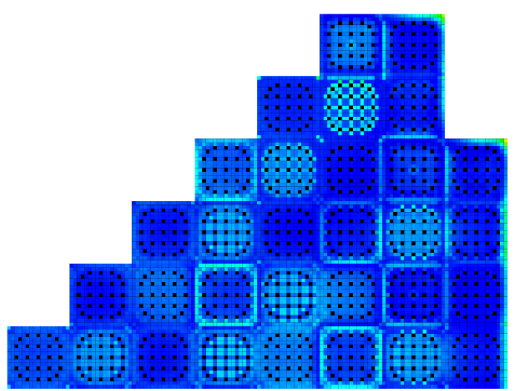

(c) DH with Refl1D

$\left(\max _{R M S}=3.26\right)$

Figure 4: RMS of power axial error per pin.

\begin{tabular}{|c|c|c|c|c|c|c|c|}
\hline Method & $k-k_{\text {ref }}$ & min & $\max$ & rms & N. global iterations & N. proc & Run-Time \\
\hline DIRECT & $k_{\text {ref }}=0.9672$ & ref & ref & ref & 30 & 1600 & $457 \mathrm{~min}$ \\
\hline DH-2D Refl & $-25 \mathrm{pcm}$ & $-5.2 \%$ & $18.0 \%$ & $1.4 \%$ & 18 & 800 & $22 \mathrm{~min}$ \\
\hline DH-1D Refl & $-20 \mathrm{pcm}$ & $-9.1 \%$ & $3.1 \%$ & $1.1 \%$ & 18 & 800 & $25 \mathrm{~min}$ \\
\hline Two-Step & $-22 \mathrm{pcm}$ & $-9.5 \%$ & $3.2 \%$ & $2.1 \%$ & 0 & 800 & $6.7 \mathrm{~min}$ \\
\hline
\end{tabular}

Table 1: Comparison of Two-Step and DH methods against reference.

Besides avoiding the infinite lattice approximation and leakage model, the DH approach has the following advantages: (i) there is no need for a flux reconstruction, since the transport solution is locally available; (ii) no need for a multiparametrized cross-sections library and, therefore, no need for interpolation of homogenized data, since the assembly transport calculation can be performed at the actual core conditions; (iii) the depletion calculation can be performed using transport fluxes at the pin-level; (iv) the possibility to homogenize the radial reflector using a heterogeneous geometry and taking into account the real environment of the core. The results are promising for further investigations such as in case of axial heterogeneities, caused for instance by the presence of partially inserted control rods, or in multiphysics coupling, or depleted assemblies at the actual core conditions. Coarser mesh homogenized per quarter of assembly using higher order polynomials for the diffusion solver will be tested as well. Further investigations will be oriented in particular for the response of the axial reflector.

\section{REFERENCES}

[1] A. Kavenoky. “The SPH homogenization method.” Procedings of a Specialists' Meeting on Homogenization Methods in Reactor Physics (1978).

[2] A. Hébert. "A Consistent Technique for the Pin-by-Pin Homogenization of a Pressurized Water Reactor Assembly." Nucl Sci Eng, volume 113(3), pp. 227-238 (1993). 
[3] K. Koebke. "A New Approach to Homogenization and Group Condensation." IAEATECDOC, (231), p. 303 (1980).

[4] K. Koebke and L. Hetzelt. "On the Reconstruction of Local Homogeneous Neutron Flux and Current Distributions of Light Water Reactors from Nodal Schemes." Nucl Sci Eng, volume 91, pp. 123-131 (1985).

[5] K. Smith. "Assembly homogenization techniques for light water reactor analysis." Progress in Nuclear Energy, volume 17(3), pp. 303 - 335 (1986).

[6] R. Sanchez. "Assembly homogenization techniques for core calculations." Progress in $\mathrm{Nu}$ clear Energy, volume 51(1), pp. 14 - 31 (2009).

[7] R. Sanchez, G. Dante, and I. Zmijarevic. "Diffusion Piecewise Homogenization via Flux Discontinuity Ratios.” Nucl Eng Technol, volume 45(6), pp. 707 - 720 (2013).

[8] F. Rahnema and E. M. Nichita. "Leakage corrected spatial (assembly) homogenization technique." Ann Nucl Energy, volume 24(6), pp. 477 - 488 (1997).

[9] F. Rahnema, , and M. Mckinley. "High-order cross-section homogenization method." Ann Nucl Energy, volume 29, pp. 875-899 (2002).

[10] T. Takeda, K. Kirimuraand, and Y. Fujita. "Leakage Dependent SPH Factor for PWR Whole Core Transport Calculation." Int Conf of the Physics of Reactors Nuclear Power: A Sustainable Resource (September 2008).

[11] P. Mondot and R. Sanchez. "An iterative homogenization technique that preserves assembly core exchanges." International conference on supercomputing in nuclear applications SNA'2003 (2003).

[12] D. Colameco, B. Ivanov, D. Beacon, and K. Ivanov. "Iterative Transport-Diffusion Methodology for LWR Core Analysis." SNA+MC 2013, volume 41 (2014).

[13] M. Grimod, R. Sanchez, and F. Damian. "A dynamic homogenization model for pebble bed reactors." Nuclear Science and Technology, volume 52, pp. 932 - 944 (2015).

[14] A. Galia, I. Zmijarevic, and R. Sanchez. "2D Core Calculation based on the method of Dynamic Homogenization.” Proceedings of M\&C 2019, Portland, OR (August 2019).

[15] T. Kozlowski and T. J. Downar. "PWR MOX/UO2 Core Transient Benchmark (Final Report)." NEA/NSC/DOC(2006)20, volume 6048 (2007).

[16] R. Sanchez, I. Zmijarevic, M. Coste-Delclaux, E. Masiello, S. Santandrea, E. Martinolli, L. Villate, N. Schwartz, and N. Guler. "APOLLO2 Year 2010." Nucl Eng Technol, volume 42(5) (October 2010).

[17] E. Masiello, R. Sanchez, and I. Zmijarevic. "New Numerical Solution with the Method of Short Characteristics for 2-D Heterogeneous Cartesian Cells in the APOLLO2 Code: Numerical Analysis and Tests." Nucl Sci Eng, volume 161(3), pp. 257-278 (2009).

[18] Y. S. Ban, E. Masiello, R. Lenain, H. G. Joo, and R. Sanchez. "Code-to-code comparisons on spatial solution capabilities and performances between nTRACER and the standalone IDT solver of APOLLO3@.” Ann Nucl Energy, volume 115, pp. 573 - 594 (2018). 